\title{
Análisis exploratorio espacial de los accidentes de tránsito en Ciudad Juárez, México
}

\author{
Vladimir Hernández Hernández ${ }^{1}$
}

Forma de citar Hernández Hernández V. Análisis exploratorio espacial de los accidentes de tránsito en Ciudad Juárez, México. Rev Panam Salud Publica. 2012;31(5):396-402.

RESUMEN Objetivo. Elaborar una herramienta para el estudio exploratorio de los accidentes viales en Ciudad Juárez, Chihuahua, México, que aplique de forma exclusiva la variable geográfica espacial (ubicación).

Método. Estudio observacional y transversal que utiliza un sistema de información geográfica para explorar la naturaleza espacial de 13305 accidentes viales registrados durante 2008 y 2009 en Ciudad Juárez. Se construyeron indicadores aproximados al flujo de tránsito y se incluyeron dos variables: indice de jerarquía urbana y densidad de población.

Resultados. El valor de la autocorrelación espacial global fue positivo, indicando la presencia de agrupamientos que fueron identificados a través de los indicadores de asociación espacial. Existen clústeres de riesgo vial localizados en las zonas con una alta jerarquía intraurbana, baja densidad de población y un elevado flujo de tránsito.

Conclusiones. El análisis exploratorio de datos espaciales es una fase previa al uso de técnicas multivariadas de mayor alcance. La aplicación de las técnicas del análisis exploratorio por sí misma permite tipificar agrupamientos espaciales, identificar autocorrelación global e indicar la dirección de las variables en estudio.

Palabras clave Accidentes de tránsito; sistemas de información geográfica; prevención de accidentes; técnicas de análisis; México.

En las Américas los accidentes de tránsito constituyen un grave problema de salud pública. En 2007, el número de personas fallecidas ascendió a 142 252, de los cuales el $39 \%$ fueron usuarios vulnerables (motociclistas, ciclistas y peatones) y tres cuartas partes fueron hombres. Del total de países de la región, durante ese año 11 se ubicaron por arriba de la tasa media regional de mortalidad $(15,8 \%)$ y, de estos, México registró unas de las tasas más altas (21,7 fallecidos por 10000 habitantes), superado solo por Venezuela con 21,8 fallecidos (1).

\footnotetext{
1 Universidad Autónoma de Ciudad Juárez, Ciudad Juárez, Chihuahua, México. La correspondencia se debe dirigir a Vladimir Hernández Hernández. Correo electrónico: vladimir.hernandez@uacj.mx
}

En 2008 México, uno de los 10 países con mayor mortalidad por accidentes de tránsito en todo el mundo, registró una tasa media de 22,5 muertes por 100000 habitantes (2). Su estado de Chihuahua, con 33,7 muertes por 100000 habitantes, no solo supera a la tasa nacional, sino que además casi duplica a la de 6,9 registrada en el Distrito Federal, capital de México y la ciudad del país con mayor densidad poblacional (3). En el caso de Ciudad Juárez (Chihuahua), dicha tasa alcanza a 7,2 y la de lesionados a 255 por 100000 habitantes (4).

Ciudad Juárez experimenta un proceso de expansión urbana que, paralela y lógicamente, trae aparejada una expansión similar del parque automotor.
Según datos del Observatorio de Seguridad y Convivencia Ciudadana del municipio, en la ciudad circulan unos 750000 vehículos, los cuales, considerando los 1332131 habitantes que notificó el censo de población de 2010, resulta en una tasa de 1,7 vehículos por habitante (4).

Esta nueva realidad local plantea la necesidad de introducir mejoras en la infraestructura y en las señalizaciones viales, así como de ampliar la cobertura y la eficiencia de los agentes de tránsito. En este sentido, es imprescindible que quienes toman decisiones cuenten con las herramientas adecuadas para llevar a cabo sus acciones con un enfoque planificado $-\mathrm{y}$ dirigido sobre todo a los lugares donde las condiciones del trán- 
sito son más delicadas- que permita dar un marcado salto cualitativo en la seguridad vial predominante en México y en sus municipios. Dado que la idea es pasar de una actitud reactiva al establecimiento de medidas preventivas, se torna perentorio trabajar con apoyo de instrumentos de análisis espacial de los sistemas de información geográfica —un componente útil, aunque no suficiente para afrontar el reto de reducir los accidentes de tránsito y las altas tasas de mortalidad que provocan.

Establecer un vínculo causal entre ciertos factores de riesgo y la incidencia de accidentes de tránsito plantea muchas dificultades generadas en la índole de las variables involucradas, entre las cuales —además del creciente parque automotor- figuran la frecuencia horaria, los niveles de empleo, el comportamiento de los viajes, el sexo, la edad y el uso de dispositivos de seguridad (5-7).

La literatura aporta valiosos conocimientos sobre la distribución de los accidentes de tránsito en el espacio geográfico, contribuyendo a identificar agrupamientos espaciales (o clústeres) y también a desarrollar métodos para elaborar estimaciones sólidas (8-14). Estos modelos, que consideran al espacio geográfico como un determinante clave para estudiar las relaciones que existen según la localización de los siniestros viales, son poco utilizados en México. Una prueba del aprovechamiento de la información geográfica para formular estrategias de prevención es la iniciativa DDACTS $^{2}$ la cual a partir de análisis espaciales y temporales contribuye a fortalecer las medidas de seguridad pública en general, ya que no solo evalúa datos sobre siniestralidad sino también sobre criminalidad $(15,16)$. Por otro lado se considera que la seguridad vial es una más de las dimensiones de la seguridad humana, y así lo apuntó el Programa de las Naciones Unidad para el Desarrollo, hace más de una década (17). Diversos autores sostienen además que entre los factores de riesgo que las personas deben enfrentar en su vida diaria destacan los relativos al tránsito automotor $(18,19)$.

El presente trabajo propone un modelo simplificado de exposición cuyo objetivo general fue elaborar una herramienta para el estudio exploratorio de

\footnotetext{
2 Siglas de Data-Driven Approaches to Crime and Traffic Safety (enfoques basados en datos sobre el crimen y la seguridad pública).
}

los accidentes viales en Ciudad Juárez que aplique de forma exclusiva la variable geográfica espacial (ubicación). Su desarrollo está enmarcado en el análisis exploratorio de datos espaciales integrados en los sistemas de información geográfica. Sus objetivos particulares incluyeron identificar agrupamientos espaciales y su nivel de significancia estadística; explorar la relación espacial entre la exposición al riesgo y la densidad poblacional y la incidencia de siniestros viales, y valorar la utilidad de las técnicas exploratorias de datos espaciales en el rubro de accidentes de tránsito.

\section{MATERIALES Y MÉTODOS}

\section{Diseño}

Se realizó un estudio de tipo observacional transversal con información sobre accidentes de tránsito, población y empleo en Ciudad Juárez. Se construyó un sistema de información geográfica (SIG) integrando una base digital clasificada en áreas geoestadísticas básicas ${ }^{3}$ (AGEB) urbanas. Para los análisis se utilizaron los programas (software) ArcView 9.3 y GeoDa —este último gratuito, desarrollado por el Centro GeoDa de análisis y computación geoespacial de la Universidad del Estado de Arizona, Estados Unidos.

\section{Recolección de datos}

La información, proporcionada por la Dirección General de Tránsito Municipal de Juárez, consistió en una base geocodificada que incluyó un total de 13305 incidentes de tránsito recopilados durante el período enero de 2008-agosto de 2009, entre ellos colisiones, atropellamientos, volcaduras, caídas de pasajeros y colisiones con objetos fijos. La base de datos ha sido producto de un proceso de cooperación conjunta entre varias entidades relacionadas con el tránsito y la seguridad pública, enmarcado en la Iniciativa Mexicana de Seguridad Vial ${ }^{4}$ (Imesevi). De este modo, se pudo traba-

\footnotetext{
3 Es la unidad censal más pequeña, que incluye entre 20 y 50 manzanas y no debe estar dividida por carreteras, aeropuertos, ríos o grandes áreas despobladas.

4 Un programa federal de prevención de accidentes de tránsito, que comenzó como un programa piloto en ocho municipios de México: Guadalajara y Zapopán en Jalisco; Monterrey y San Pedro Garza en Nuevo León; Celaya y León en Guanajuato, y Gustavo A. Madero y Cuauhtémoc en el Distrito Federal. En Ciudad Juárez se inició en 2008.
}

jar con informes y otros documentos de verificación sólidos y confiables.

La base de datos está geocodificada a nivel de intersección, pero para poder correlacionarla con las otras variables del estudio se agregó la información en las unidades censales más pequeñas. No se analizó información de tipo temporal, lo cual podría considerarse una limitación de este trabajo.

\section{Análisis exploratorio}

Se realizó un análisis exploratorio de datos espaciales (AEDE) que consiste en una serie de técnicas para estimar y visualizar la autocorrelación espacial. El AEDE permite observar cómo se distribuye la siniestralidad vial en el ámbito urbano e identificar la presencia de clústeres de riesgo vial, es decir zonas de la ciudad donde se concentran los accidentes de tránsito.

Una manera de cuantificar dicha tendencia es a través del índice I de Morán, un indicador de autocorrelación que mide precisamente la tendencia de los valores a agruparse o no en el espacio. Para calcular el nivel de agrupamiento se define una matriz de pesos geográficos, la cual determinará qué unidades son vecinas. Una forma de visualizar la relación es a través de un diagrama de dispersión, con un recorrido de $-1 \mathrm{a}+1$, donde el extremo negativo indica ausencia de autocorrelación, en constraste con el extremo positivo, que indica que las unidades vecinas son muy parecidas entre sí (20-22).

Otra técnica más intuitiva y específica de la presencia de clústeres es facilitada por los indicadores locales de asociación espacial. Este indicador considera el valor que registran los valores vecinos y los compara por medio de pruebas de hipótesis para determinar sus grados de agrupamiento (20). A diferencia del I de Morán, estos agrupamientos sí se pueden representar en el espacio, por ejemplo clasificando los datos en unidades administrativas censales $-\mathrm{O}$ cualquier otra forma espacial - y visualizando los resultados en un mapa.

\section{Análisis con variables aproximadas}

Debido a que los datos disponibles en las instituciones mexicanas de planificación, y en particular las dedicadas al transporte, por lo general no reflejan de forma directa los flujos vehiculares, 
para el presente estudio se decidió por lo tanto utilizar variables aproximadas, un enfoque que ya ha sido empleado en otros trabajos $(23,24)$. La ventaja de este enfoque es la mayor disponibilidad de información, toda vez que requiere datos de población y de empleo, ambos suficientemente recogidos en censos y conteos publicados en México.

Las variables aproximadas se denominaron: población próxima y empleo próximo, ambas buscando captar el efecto del flujo de tránsito de manera indirecta. El índice de jerarquía intraurbana es otra de las variables aproximadas, en este caso, como un indicador del uso de suelo comercial y de servicios. A las anteriores se suma la proporción de accidentes de tránsito y la densidad de población, que son las variables del análisis.

Se partió del supuesto de que el volumen de tránsito que genera una zona está en proporción con el nivel de empleo (E) y con la población $(\mathrm{P})$ residente en cada zona. Por lo tanto, el empleo próximo (EP) y la población próxima (PP) se construyen para capturar el efecto del tránsito y se espera que el volumen de tránsito se incremente a partir de los mayores niveles de empleo y de población residente en una determinada zona comparada con otras vecinas. Para los viajes generados por las zonas se construyeron los siguientes indicadores:

$$
E P i=\sum_{i}^{i \neq j} \frac{E j}{d i j} \quad P P i=\sum_{i}^{i \neq j} \frac{P j}{d \ddot{j}}
$$

Donde Ej es el empleo total; $P j$ es la población total y dij es la distancia entre la zona $i$ y la $j$.

\section{Análisis del uso de suelo}

El uso de suelo terciario - definido por el predominio de actividades comerciales y de servicios- tiene un impacto directo en el número de accidentes de tránsito debido a que genera un mayor número de viajes vehiculares $(23,24)$. Dado que para el presente estudio no se contó con información desagregada de los usos de suelo o con una capa de información digital, en su lugar se construyó un índice de jerarquía urbana con datos sobre empleo a nivel de AGEB del censo económico de 2004 y de población del Conteo 2005 (25). El censo económico se lleva a cabo regularmente cada cinco años y capta la información de todas las unidades económicas denominadas establecimientos. Para las localidades urbanas, como Ciudad Juárez, recoge la información de las actividades de comercio y servicios no financieros. El conteo de población también se lleva a cabo cada cinco años, generalmente a la mitad del período intercensal, es decir, se levanta entre censos. En su versión 2005, el conteo capturó dos dimensiones: características de la población y de la vivienda. Con los datos del total de empleo en comercio y servicios y de población total se elaboró el siguiente indicador:

$$
C i j=\frac{\left(\frac{E i j}{P j}\right)}{\left(\frac{E i}{P}\right)}
$$

Donde Cij es el grado de centralidad de cierta actividad $i$ en determinada zona $j$; Eij trabajadores de la actividad $i$ en el zona $j$; $P j$ es la población de la zona $j$, y $P$ es la población total de la ciudad.

\section{RESULTADOS}

Se analizaron 599 unidades censales con datos de accidentes de tránsito y los indicadores propuestos. La selección de las técnicas resultó consistente para determinar el nivel de agrupamientos y de correlación entre las variables estudiadas. Uno de los primeros resultados se puede ver en la figura 1, donde la proporción de valores bajos de accidentes de tránsito está representada por los tonos claros distribuidos mayoritariamente en el extremo poniente de la ciudad. En con-

\section{FIGURA 1. Distribución geográfica de accidentes de tránsito, Ciudad Juárez, Chihuahua, México, 2008-2009}
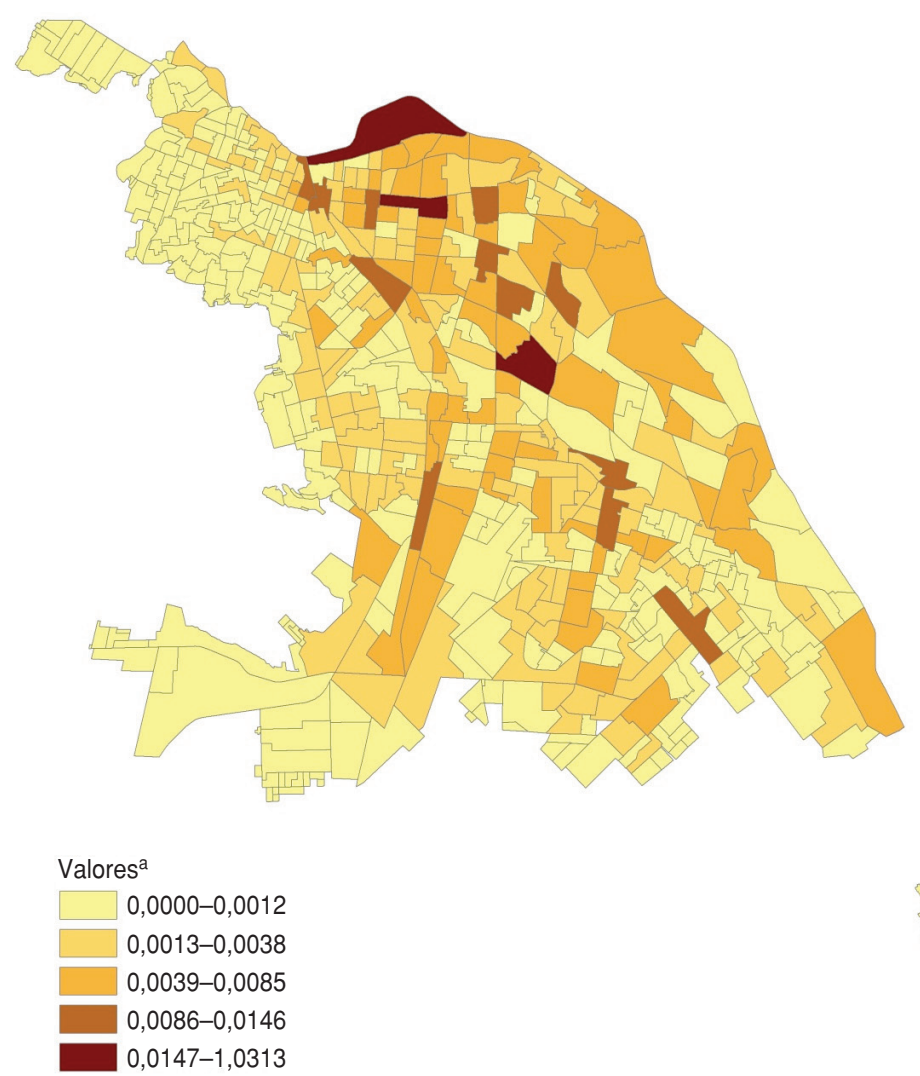

Fuente: elaboración del autor, con datos de la Dirección General de Tránsito Municipal, Ciudad Juárez, Chihuahua, México.

Nota: Los valores de los ejes son distancias ponderadas de accidentes de tránsito y una matriz de pesos espaciales - distancia euclidiana - entre las observaciones. El cuadrante superior derecho ubica a las áreas geoestadísticas básicas (AGEB) con valores elevados de accidentes de tránsito, lo que favorece al valor positivo del I de Morán, mientras que el cuadrante inferior izquierdo se relaciona con AGEB de valores bajos. Los cuadrantes superior izquierdo e inferior derechos tienen valores combinados bajos-altos de accidentes de tránsito.

a Proporción de accidentes de tránsito (total de accidentes de tránsito/total de accidentes de tránsito en la ciudad) 
FIGURA 2. Autocorrelación espacial entre accidentes de tránsito, Ciudad
Juárez, Chihuahua, México, 2008-2009

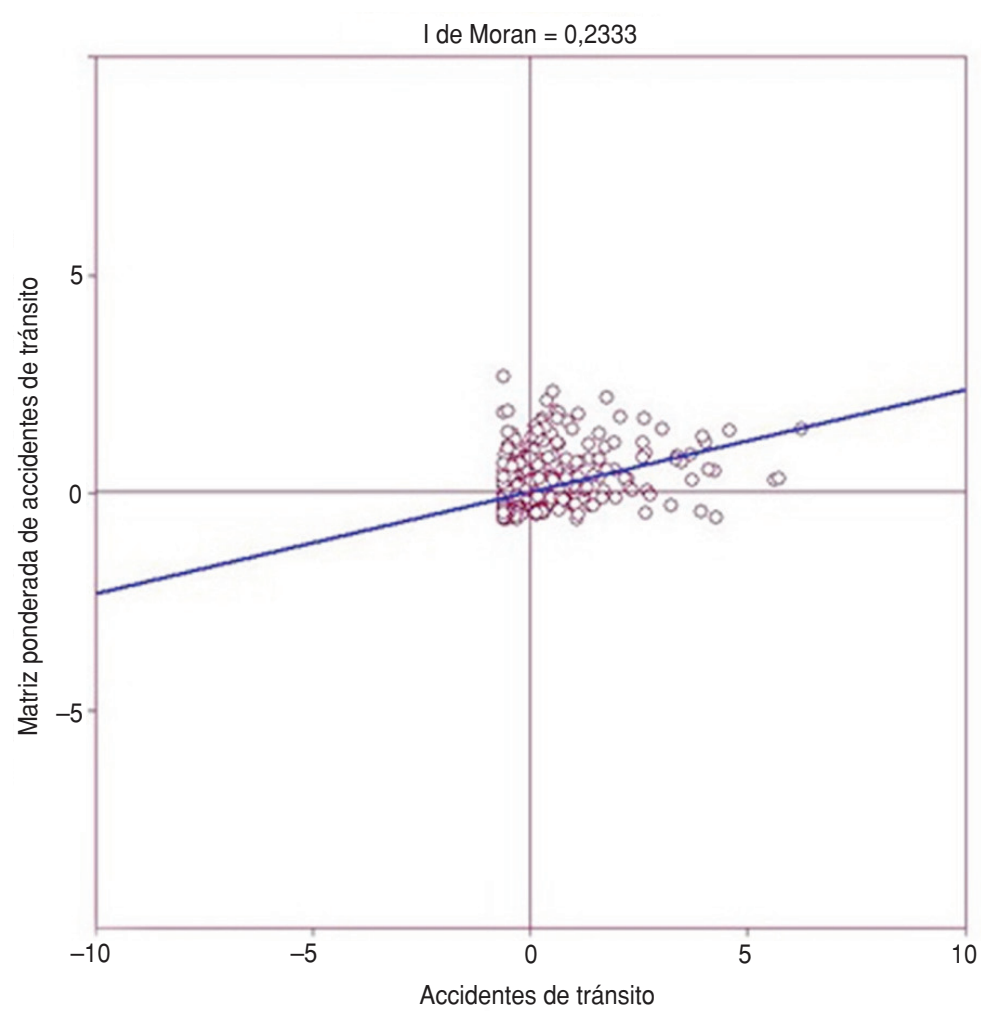

Fuente: elaboración del autor, con datos de la Dirección General de Tránsito Municipal, Ciudad Juárez, Chihuahua, México.

traste, la zona de la ciudad con el mayor desarrollo urbano coincide con los tonos oscuros (alta proporción de accidentes). Se observa así un claro patrón espacial que divide a la ciudad en dos secciones.

Este panorama concuerda con estudios previos que mencionan la agrupación de estos eventos en determinadas zonas de las ciudades (8-10, 14, 23, 24). Sugiere además que al menos en Ciudad Juárez el patrón espacial registra los valores más bajos en las orillas de la ciudad, particularmente al oeste, mientras que se observa una alta proporción en zonas del centro-norte y valores medios en la zona sur-oriente.

El valor del índice I de Morán indica la presencia de autocorrelación espacial positiva 0,2333 con un nivel de significancia de $P<0,05$, es decir que existe una significativa aglomeración de zonas con similares valores de siniestros viales. La figura 2 muestra que las áreas con altos valores en la proporción de siniestros viales (cuadrante superior derecho del gráfico) tienden a agruparse, contribuyendo a la autocorrelación espacial positiva. En la figura 3, los clústeres rojos y azules indican donde es estadísticamente significativo $(P<0,05)$ identificar agrupamientos con valores similares. Las zonas con altos valores de siniestros viales forman un clúster central que se extiende desde el centro histórico de la ciudad hacia el oriente a la zona conocida como Programa Nacional Fronterizo (PRONAF), mientras que las áreas azules representan clústeres estadísticamente significativos con bajos niveles de siniestralidad vial. Los tonos azules y rojos pálidos representan zonas donde los valores son disímiles (altos-bajos y bajosaltos). En consecuencia, los indicadores locales pueden auxiliar al investigador o al tomador de decisiones identificando zonas de alto o bajo riesgo vial, así como la presencia de áreas disimiles.

La medida del índice I de Morán para la variable de siniestros viales (positiva y significativa) sugiere la presencia de autocorrelación espacial. Sin embargo, provee una mirada parcial del fenómeno analizado, ya que el valor del clúster alto-alto refleja, por ejemplo, la contribución que realizan variables como la densidad de población, el índice de jerarquía intraurbana y las variables aproximadas al flujo de tránsito. En teoría, hay otras variables o dimensiones que influyen en la frecuencia de los accidentes de tránsito, pero considerando exclusivamente la naturaleza espacial de los fenómenos se está ante la presencia de zonas con un efecto de "contagio espacial", es decir, ante la probabilidad de que zonas con altos valores de siniestros viales se ubiquen en la vecindad de zonas que también registran tasas altas de accidentes de tránsito. Las correlaciones entre la variable accidentes de tránsito y los indicadores aproximados -flujo vial, uso de suelo comercio y servicios y densidad de población- arrojaron diferentes valores, todos estadísticamente significativos (cuadro 1).

En cuanto a la dirección de la correlación, como se ve en la figura 4, la pendiente entre densidad de población y accidentes de tránsito fue negativa (B), en tanto que las tres restantes correlaciones (A, C y D) fueron positivas. Los resultados son consistentes con estudios previos (23, 24) que observaron una menor proporción de accidentes de tránsito en zonas de la ciudad con baja densidad de población y mayor proporción de accidentes en las zonas dedicadas al comercio y los servicios. Como suele ocurrir, los indicadores usados en este trabajo ofrecen ventajas y desventajas. Lo que los hace adecuados es su disponibilidad y su integración en una herramienta de análisis.

\section{DISCUSIÓN}

El análisis exploratorio fue apropiado para evaluar, como primer paso, la naturaleza geográfica de los datos sobre accidentes de tránsito en Ciudad Juárez, mientras que los indicadores fueron adecuados para caracterizar medidas aproximadas a la exposición y son citados en la literatura sobre el tema $(6,23,24)$.

Los métodos de análisis geoestadísticos están desarrollándose rápidamente en años recientes. Más allá de las limitaciones que se puedan presentar en la disponibilidad de datos, la experiencia del presente trabajo permite mencionar dos posturas respecto a dichos métodos. Una es que las bases cartográficas de siniestros viales, dado que suelen estar puntualmente geo-referenciadas, tienen la ventaja de determinar localizaciones muy cercanas a la ideal o exacta del fenómeno -favoreciendo el análisis geoestadístico $(9,10)$. La segunda postura indica que 
FIGURA 3. Indicadores locales de asociación espacial para los accidentes de tránsito, Ciudad Juárez, Chihuahua, México, 2008-2009
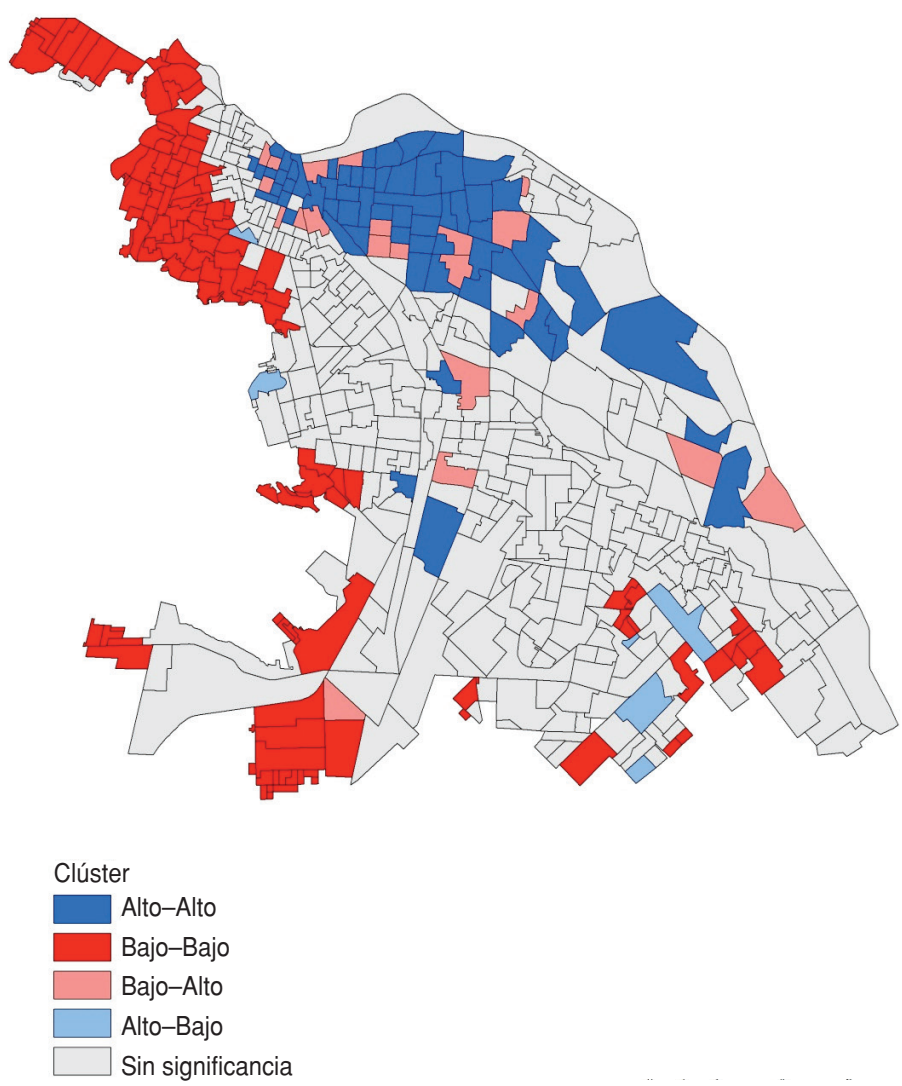

Fuente: elaboración del autor, con datos de la Dirección General de Tránsito Municipal, Ciudad Juárez, Chihuahua, México.

CUADRO 1. Correlación entre accidentes de tránsito e indicadores aproximados, Ciudad Juárez, Chihuahua, México, 2008-2009

\begin{tabular}{lc}
\hline \multicolumn{1}{c}{ Variable } & Correlación $^{a}$ \\
\hline Empleo próximo & 0,668 \\
Población próxima & 0,726 \\
Índice de centralidad & \\
$\quad$ (uso de suelo comercio y servicio) & 0,083 \\
Densidad de población & $-0,088$ \\
\hline
\end{tabular}

Fuente: Elaboración del autor, con datos de la Dirección General de Tránsito Municipal, Ciudad Juárez, Chihuahua, México, y cálculos de la GeoDa, Arizona, Estados Unidos.

a Significancia estadística $P<0,05$.

para correlacionar los datos socioeconómicos - generalmente agregados en zonas administrativas- con los accidentes de tránsito es necesario agrupar a los accidentes. Sin bien este procedimiento tiene la desventaja de que pierde información puntual, tiene la ventaja de que permite avanzar en la búsqueda de asociaciones entre las variables que el investigador desee explorar $(23,24)$.

De este acercamiento exploratorio se han podido extraer indicios significa- tivos de la relación y direccionalidad de las variables analizadas, una condición muy importante para poder llevar a cabo un sólido análisis multivariante. Hipotéticamente, el hecho de registrar autocorrelación espacial debe sugerir su incorporación, por ejemplo, en modelos multivariados de regresión espacial. Lo que ofrece el análisis exploratorio de datos espaciales es un camino para determinar qué modelo se necesita poner a prueba, en particular el programa
GeoDa, que brinda una serie de diagnósticos de tipo espacial que permiten determinar qué tipo de autocorrelación ${ }^{5}$ está presente en los datos $\mathrm{y}$, en consecuencia, qué modelo se ha de utilizar. Adicionalmente, estos métodos de análisis pueden efectuarse con un mínimo de información, con un equipo de cómputo modesto y con un programa de acceso gratuito, percibiéndose entonces como un valioso insumo que equilibre el constante aumento de las demandas de mayor seguridad vial por parte de los ciudadanos.

Décadas de investigación para el diseño, el manejo y la aplicación de los SIG han conducido a los estudios sobre accidentes de tránsito hacia la adopción de un enfoque espacial $(15,16)$. Uno de los retos consiste en acercar estas herramientas a los encargados directos de proveer mayor seguridad vial, quienes en muchos de los casos siguen basándose en la intuición y la experiencia como principales fuentes de información y otros insumos. El modelo del presente estudio tiene un diseño sencillo, se aplica fácilmente y arroja resultados inmediatos.

\section{Conclusiones}

El análisis espacial a partir de los datos de accidentes de tránsito y las estadísticas de empleo y población por unidad espacial administrativa (AGEB) permitió estimar los niveles de dependencia espacial y demostró la pertinencia de efectuar un estudio preliminar de los datos. La exploración de los datos constituye una contribución para las autoridades de tránsito y de seguridad pública, quienes son las principales beneficiadas con el análisis exploratorio porque incrementan sus recursos operativos, favoreciendo así el equilibrio entre el aumento de las exigencias en su trabajo y el correspondiente incremento de su capacidad de gestión. Asimismo, la aplicación de estas técnicas por las oficinas municipales promueve la concentración de recursos en las zonas

\footnotetext{
5 A grandes rasgos hay dos tipos de autocorrelación. El primer ejemplo de autocorrelación está presente en el término del error y viola el supuesto de la no correlación de los errores, uno de los supuestos de método de regresión por mínimos cuadrados ordinarios. En este caso se puede solucionar proponiendo un modelo espacial del error. El segundo tipo se presenta cuando el valor de la variable dependiente está determinado por el valor de la variable en las AGEB vecinas. En este caso, la alternativa es proponer un modelo espacial lag (una relación interactiva entre la variables de AGEB vecinas).
} 
FIGURA 4. Dispersión entre accidentes de tránsito, índice de centralidad, densidad poblacional, empleo próximo y población próxima, Ciudad Juárez, Chihuahua, México, 2008-2009
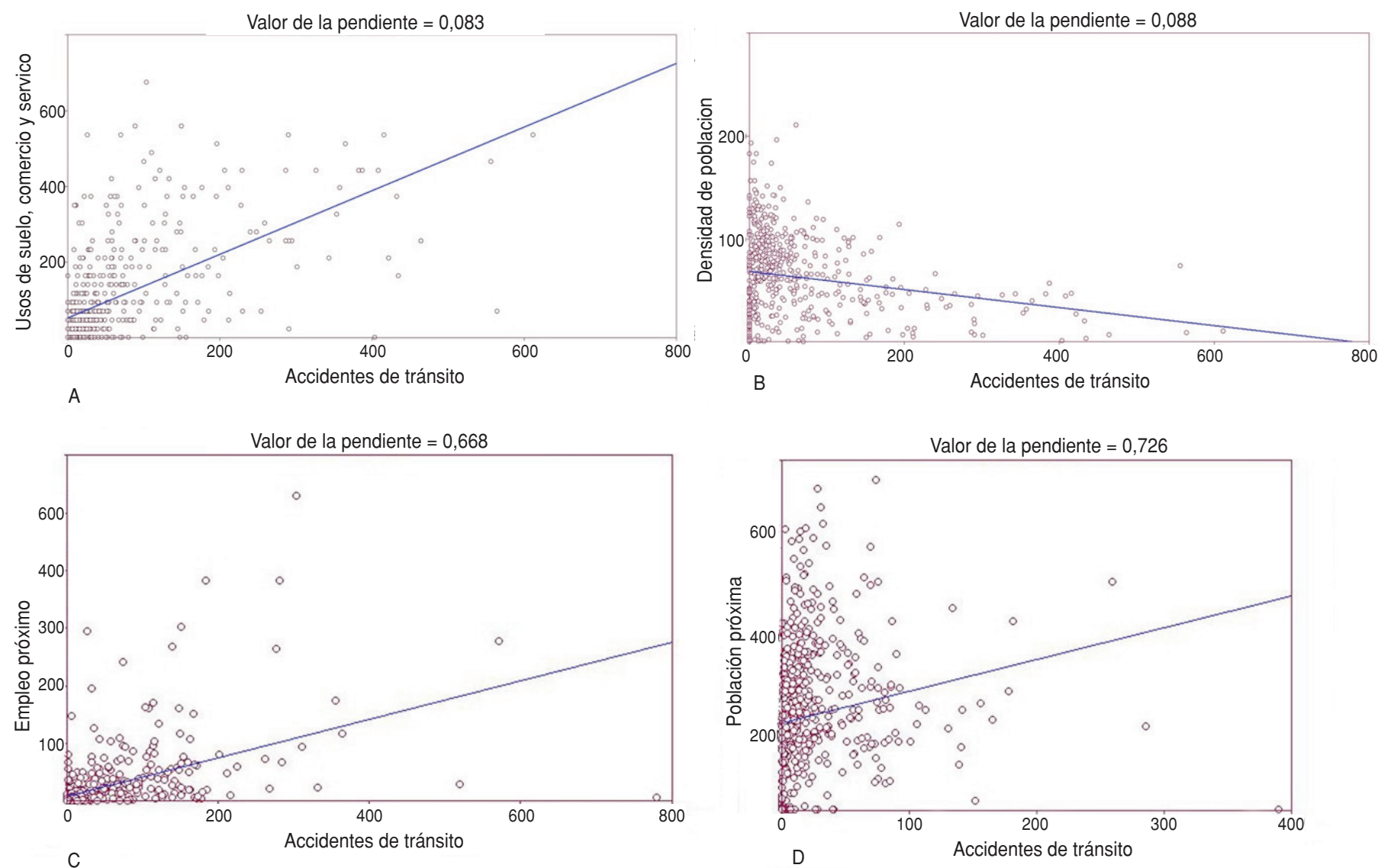

Fuente: elaboración del autor, con datos de la Dirección General de Tránsito Municipal, Ciudad Juárez, Chihuahua, México.

Nota: Las gráficas A, B, C y D representan (en el eje horizontal) el valor de los accidentes de tránsito por áreas geoestadísticas básicas (AGEB) y (en el eje vertical) los valores de las variables aproximadas "usos de suelo terciario (comercio y servicios), empleo próximo, población próxima y densidad de población" por AGEB.

conflictivas y da la posibilidad de incluir datos de problemas que afectan a la seguridad de las personas. La disponibilidad de GeoDa (un software abierto) es un recurso que podría ser ampliamente aprovechado no solo por las entidades de seguridad pública sino también por otras oficinas públicas de México a nivel federal, estatal y municipal. La disponibi-

1. Organización Panamericana de la Salud. Informe sobre el estado de la seguridad vial en la Región de las Américas. Washington, D.C.: OPS; 2009

2. Organización Mundial de la Salud. Informe sobre la situación de la seguridad vial: es hora de pasar a la acción. Ginebra, Suiza: OMS; 2009.

3. Centro Nacional de Prevención de Accidentes. Perfil accidentes de tránsito. República Mexicana, Observatorio Nacional de Lesiones. Dis- lidad de GeoDa y los datos utilizados en el análisis espacial tienen el potencial de poder ser ampliamente utilizados por los países de la Región.

Las variables aproximadas y los datos de accidentes de tránsito favorecen la identificación de zonas de riesgo y la posibilidad de aplicar medidas de bajo costo para reducir las tasas de mortalidad

\section{REFERENCIAS}

ponible en: http://www.cenapra.salud.gob. mx/CENAPRA_2010/estadisticas/Perfil Nacional_2008.pdf Acceso el 5 de febrero de 2012.

4. Observatorio de Seguridad y Convivencia Ciudadana del Municipio de Juárez, Chihuahua. Incidentes de tránsito 2007-2009. Disponible en: http://www.observatorio dejuarez.com/Estadísticas/Incidentesde tránsito20072009/tabid/458/Default.aspx Acceso el 5 de febrero de 2012. y de lesionados. Se concluye que el análisis exploratorio de datos espaciales es útil para esbozar el nivel de correlación y direccionalidad entre dos variables, identificar y delimitar zonas con altos valores de accidentes de tránsito, poner en práctica un enfoque preventivo y constituirse como una herramienta de apoyo para las acciones de seguridad vial.
5. Kim JK, Ulfarsson GF, Shankar VN, Kim S. Age and pedestrian injury severity in motorvehicle crashes: a heteroskedastic logit analysis. Accid Anal Prev. 2008;40(5):1695-702

6. Wier M, Weintraub J, Humphreys EH, Seto E, Bhatia R. An area-level model of vehiclepedestrian injury collisions with implications for land use and transportation planning. Accid Anal Prev. 2009;41(1):137-45.

7. Donate-López C, Espigares-Rodríguez E, Jiménez-Moleón JJ, Luna-del-Castillo J de D, 
Bueno-Cavanillas A, Lardelli-Claret P. The association of age, sex and helmet use with the risk of death for occupants of two-wheeled motor vehicles involved in traffic crashes in Spain. Accid Anal Prev. 2010;42(1):297-306.

8. Cheng W, Washington SP. Experimental evaluation of hotspot identification methods. Accid Anal Prev. 2005;37(5):870-81.

9. Loo BP. Validating crash locations for quantitative spatial analysis: a GIS-based approach. Accid Anal Prev. 2006;38(5):879-86.

10. Miaou SP, Soong JJ, Mallick BK. Roadway traffic crash mapping: A space-time modeling approach. Journal of Transportation and Statistics. 2003;6(1):33-57.

11. Mitchell A. The ESRI guide to GIS analysis, Volume 2: Spatial Measurements and Statistics. Redlands, USA: ESRI Press; 2009.

12. Steenberghen T, Dufays T, Thomas I, Flahaut B. Intra-urban location and clustering of road accidents using: GIS: a Belgian example. Int J Geogr Inf Sci. 2004;18(2):169-81.

13. Okabe A, Satoh T, Sugihara K. A kernel density estimation method for networks, its computational method and GIS-based tool. Int J Geogr Inf Sci. 2009;23(1):7-32.

14. Montella A. A comparative analysis of hotspot identification methods. Accid Anal Prev. 2010;42:571-81.
15. National Highway Traffic Safety Administration. Data-Driven Approaches to Crime and Traffic Safety (DDACTS). Operational Guidelines. Disponible en: http://stko.maryland. gov/LinkClick.aspx?fileticket=JJZrzmHIX7M \%3d\&tabid=131\&.pdf Acceso el 6 de febrero de 2012.

16. Worden RE, McLean SJ. DDACTS in Theory and Practice. The John F. Finn Institute for Public Safety, Inc. Disponible en: http:/ /stko. maryland.gov/LinkClick.aspx?fileticket $=3 \mathrm{kX}$ 1XOa2QEc\%3d\&tabid=132\&.pdf Acceso el 6 de febrero de 2012.

17. Programa de las Naciones Unidas para el Desarrollo. Informe sobre desarrollo humano 1994. Nueva York: Fondo de Cultura Económica, S.A. de C.V.; 1994.

18. Ballbé Malloll M. Seguridad integral. Un nuevo concepto. Madrid, España: Instituto Superior de Estudios de Seguridad; 2003.

19. Fernández JP. Seguridad humana. Barcelona, España: Universidad Autónoma de Barcelona; 2006. [Tesis doctoral].

20. Anselin L. Local Indicators of Spatial Association-LISA. Geogr Anal. 1995;27(2):93-115.

21. Anselin L. Interactive Techniques and Exploratory Spatial Data Analysis. En: Longley PA, Goodchild MF, Maguire DJ, Rhind DW, eds. Geographical Information Systems: Prin- ciples, Techniques, Management and Applications. New York; Wiley: 1999. Pp. 251-64.

22. Anselin L, Ibnu S, Youngihn K. GeoDa: An Introduction to Spatial Data Analysis. Geogr Anal. 2006;38(1):5-22.

23. Graham DJ, Glaister S. Spatial Variation in Road Pedestrian Casualties: The Role of Urban Scale, Density and Land-use Mix. Urban Studies. 2003;40(8):1591-607.

24. Fuentes Flores CM, Hernández V. La estructura espacial urbana y la incidencia de accidentes de tránsito en Tijuana, Baja California (2003-2004). Frontera Norte. 2009;21(42): 109-38.

25. Fuentes Flores CM. Los cambios en la estructura intraurbana de Ciudad Juárez, Chihuahua, de monocéntrica a multicéntrica. Frontera Norte. 2001;13(25):95-118.

Manuscrito recibido el 2 de septiembre de 2011. Aceptado para publicación, el 19 de diciembre de 2011.

ABSTRACT Objective. Prepare a tool for the exploratory study of road accidents in Ciudad Juarez, Chihuahua, Mexico, that exclusively applies the spatial geographical variable (location).

\section{Spatial exploratory analysis of road accidents in Ciudad Juarez, Mexico}

Key words
Methods. Observational and cross-sectional study that uses a Geographic Information System to explore the spatial nature of 13305 road accidents recorded during 2008 and 2009 in Ciudad Juarez. Indicators were constructed that approximated the transit flow and included two variables: indices of the level of urbanization and population density.

Results. The value of the global spatial autocorrelation was positive, indicating the presence of groupings that were identified through the spatial association indicators. There are road risk clusters located in areas with a high level of urbanization, low population density, and a high transit flow level.

Conclusions. The exploratory analysis of spatial data is a phase that precedes the use of multivariate techniques with a broader scope. The application of exploratory analysis techniques in itself makes it possible to standardize spatial groupings, identify global autocorrelation, and indicate the direction of the variables under study.

Accidents, traffic; geographic information systems; accident prevention; analytical methods; Mexico. 